\title{
NACHRUF / OBITUARY
}

\section{Trauer um Prof. Dr. Manfred Weiß}

\section{In Memoriam Prof. Dr. Manfred Weiß}

Am 5. März 2019 verstarb mit 76 Jahren Manfred Weiß. Mit ihm verliert die ökonomische Bildungsforschung in Deutschland einen der profiliertesten Wissenschaftler. Er arbeitete während seines gesamten Berufslebens am Deutschen Institut für Internationale Pädagogische Forschung (DIPF) in Frankfurt am Main (jetzt: DIPF | Leibniz-Institut für Bildungsforschung und Bildungsinformation). Die dortige Abteilung für Bildungsökonomie war der Ort, an dem er nach einem Studium der Betriebswirtschaft in Frankfurt 1970 eine adäquate Beschäftigung fand. Die für Friedrich Edding gegründete und - nach dessen Berufung an das Max-PlanckInstitut für Bildungsforschung in Berlin - von Hasso von Recum weitergeführte Abteilung befand sich im DIPF in einem interdisziplinären Umfeld. Vergleichende Erziehungswissenschaft, Psychologie, Bildungssoziologie, Berufsbildungsforschung, Empirische Forschungsmethoden und Bildungsrecht waren die anderen Arbeitsbereiche des Instituts, mit denen er stets in Kontakt war. Die unterschiedlichen theoretischen und methodischen Zugänge zu Fragen der Bildung und Erziehung am DIPF führten bei ihm zu einer Ausrichtung der ökonomischen Bildungsforschung, die davon bestimmt war, Problemstellungen im Bildungs- und insbesondere Schulwesen aus ökonomischer Perspektive zu untersuchen und nicht Fragestellungen und Konzepte der Ökonomie auf das Bildungswesen anzuwenden. Dies war das Spezifikum seines Forschungsansatzes und nicht selten der Ausgangspunkt kritischer Stellungnahmen zu den Analysen anderer Ökonom*innen, die den Spezifika des Schulwesens bei ihren Studien aus seiner Sicht nicht gerecht wurden. Beispielhaft dafür ist seine zusammen mit Gundel Schümer 2008 erstellte Analyse Bildungsökonomie und Qualität der Schulbildung. Kommentar zur bildungsökonomischen Auswertung von Daten aus internationalen Schulleistungsstudien.

Durch die betriebswirtschaftliche Ausbildung standen am Anfang der Forschungsarbeiten von Manfred Weiß Fragen der Kosten und Effizienz im Schulwesen. 1981 promovierte er an der Technischen Universität Berlin mit einer Dissertation zum Thema Effizienzforschung im Bildungsbereich. Auch in vielen weiteren Arbeiten ging er der Frage nach, wie das Verhältnis von Aufwand und Ertrag methodisch angemessen untersucht und bewertet werden kann, um unter der Bedingung von Knappheit 
die vorteilhafteste Alternative auszuwählen. Bis heute stellen diese Überlegungen noch eine Herausforderung für die Evaluation von Innovationen dar, weil sie kaum Berücksichtigung finden.

Die internationale Ausrichtung der Forschung am DIPF führte bei Manfred Weiß schon in seinen ersten Publikationen zur Beschäftigung mit Innovationen im Ausland, insbesondere im amerikanischen Schulwesen. In zahlreichen Artikeln hat er einerseits auf Reformansätze im amerikanischen Schulwesen (z. B. Magnetschulen) hingewiesen und sie in Deutschland bekannt gemacht, sie andererseits aber auch kritisch hinterfragt. Vor allem die ökonomischen Begründungen für einen Ausbau des Privatschulangebots in der amerikanischen Forschung haben seine Kritik herausgefordert. Mit seinen Arbeiten über die internationale Forschung zur Klassengröße hat er wichtige Impulse für die deutsche Debatte dieser Frage seit Mitte der 1980er-Jahre gegeben, als durch stark sinkende Schülerzahlen die Klassengrößen immer weiter gesenkt wurden, ohne nach den ökonomischen Implikationen und einer sinnvolleren Ressourcenverwendung (gezielter Mitteleinsatz in Klassen mit vielen Ausländerkindern etc.) zu fragen.

Nach dem Ende der gesamtstaatlichen Bildungsplanung im Jahr 1981 rückten Fragen der Bildungsfinanzierung zunehmend in das Interesse der Forschung von Manfred Weiß. Sowohl die Auseinandersetzung mit Ausgabenentwicklungen als auch mit Konzepten eines effizienten Mitteleinsatzes waren Gegenstand seiner Forschung. Mit dem Aufkommen der sogenannten Ansätze „Neuer Steuerung“ wurden für ihn ergänzend Fragen der Schul- und Unterrichtsqualität zu einem weiteren Bezugspunkt seiner ökonomischen Analysen. In den letzten Jahren konzentrierten sie sich auf die privaten Schulen, zu denen er mehrere Studien erarbeitete.

Manfred Weiß hatte zahlreiche internationale Kontakte. 1987 nahm er eine Vertretungsprofessur für Bildungsökonomie an der State University of Buffalo an der Faculty of Educational Studies an. Dadurch erhielt er vielfältige Anregungen für seine international vergleichenden Analysen. Auf der Grundlage seiner wissenschaftlichen Leistungen wurde er 1998 an der Erziehungswissenschaftlichen Fakultät der Pädagogischen Hochschule (später Universität) Erfurt zum Honorarprofessor für Bildungsökonomie und Bildungsforschung ernannt. Wegen seiner speziellen fachlichen Expertise war er auch an anderen Hochschulen als Dozent gefragt und übernahm Lehraufträge an den Universitäten Bern, Bochum, Frankfurt am Main, Mannheim (1999-2008 regelmäßig) und Zürich.

Manfred Weiß war Mitglied in mehreren wissenschaftlichen Beiräten von größeren Projekten. Besonders erwähnenswert ist seine Mitgliedschaft im ersten nationalen PISA-Konsortium. In diesem Zusammenhang hat er an mehreren Ergebnisberichten mitgearbeitet. Im Anschluss an die erste PISA-Untersuchung beteiligte er sich an einer umfangreichen internationalen Vergleichsuntersuchung des DIPF zu einigen erfolg- 
reichen PISA-Teilnehmerstaaten. Er war Mitglied der Autorengruppe, die 2003 einen ersten Bildungsbericht für Deutschland im Auftrag der KMK erstellte, und Mitglied des Konsortiums Bildungsberichterstattung, das 2006 den ersten von BMBF und KMK gemeinsam in Auftrag gegebenen Bericht Bildung in Deutschland erarbeitete.

Mehrere Zeitschriften beriefen ihn in ihren wissenschaftlichen Beirat, allen voran Die Deutsche Schule - DDS, deren Beirat Manfred Weiß seit 2008 angehörte. Weitere Zeitschriften sind die Schweizerische Zeitschrift für Bildungswissenschaften und Leadership and Policy in Schools.

Auch unter den Bildungsökonominnen war Manfred Weiß geschätzt. Er war lange Jahre Mitglied des Ausschusses für Bildungsökonomie im Verein für Socialpolitik und von 2003 bis 2005 dessen Vorsitzender. Bereits von 1997 bis 2000 war er Vorsitzender des Netzwerkes „Economics of Education“ in der European Educational Research Association. Seit 2010 war er Mitglied im Hochschulrat der Justus-Liebig-Universität Gießen.

Eine Würdigung von Manfred Weiß als Wissenschaftler darf nicht den Menschen unberücksichtigt lassen. Er war in der Sache stets engagiert und ein allseits geschätzter, humorvoller und zu Bonmots aufgelegter Kollege. Nicht zu vergessen sind aber auch seine Leidenschaft für den Jazz und sein Können als Schlagzeuger, das ihn mit vielen bekannten Musikerinnen der Jazzszene in Frankfurt am Main und darüber hinaus zusammenführte.

Das wissenschaftliche CEuvre von Manfred Weiß umfasst mehr als 200 Publikationen. Im Blick auf seine wissenschaftliche Leistung muss man sich fragen, wer die Lücke schließen soll, die er hinterlassen hat. Bildungsforscher*innen seines Zuschnitts gibt es nicht mehr in der jüngeren Generation, auch wenn die Problemstellungen, mit denen er sich befasste, weiterhin aktuell sind. Umso wichtiger ist es, sich mit seinen Beiträgen zu einer anwendungs- und auf politisches Handeln bezogenen Forschung weiterhin zu beschäftigen.

Horst Weishaupt, Frankfurt am Main

https://doi.org/10.31244/dds.2019.02.01 